\title{
En los pacientes con depresión mayor el tratamiento de activación conductual es tan eficaz como la farmacoterapia con paroxetina y superior a la terapia cognitiva
}

Dimidjian et al. J Consult Clin Psychol. 2006; 74 (4): 658-670.

\section{Objetivo}

Comparar la eficacia del tratamiento de activación conductua (TAC) con la terapia cognitiva (TC) y la farmacoterapia con paroxetina en pacientes con depresión mayor (DM).

\section{Diseño}

Ensayo clínico, aleatorizado y controlado

Lugar

\section{Pacientes}

241 adultos con DM según los criterios del Manual de Diagnóstico y Estadísticas de Salud Mental con puntaje mayor a 20 en el Inventario de Beck (ID) y a 14 en la escala de Hamilton (EH).

\section{Intervención}

Fueron asignados 45 pacientes a 24 sesiones de 50 minutos durante 16 semanas de TC; 43 a similar agenda de sesiones de TAC; 100 a farmacoterapia con paroxetina (10mg diarios aumentándose progresivamente la dosis hasta llegar a $50 \mathrm{mg} / \mathrm{día}$ en la semana 12) y 53 a placebo.

\section{Medición de resultados principales}

El análisis se hizo por intención de tratar y el resultado principal se midió por la variación en el puntaje de las escalas IB y EH desde el valor basal hasta los de las semanas 8 y 16 .

\section{Resultados principales}

Previamente al inicio del tratamiento los cuatro grupos eran de severidad equivalente (similar puntaje en las escalas). En los pacientes con DM severa, el promedio de los puntajes en los grupos sometidos a TAC y a paroxetina mostró una importante pero similar disminución a la semana 16 respecto de los puntajes al inicio del tratamiento. Esta mejoría fue superior a la alcanzada con TC.

Tabla 1: puntajes de las escalas a las 16 semanas en pacientes con depresión severa (menor puntaje implica mejor estado de ánimo).

\begin{tabular}{|c|c|c|c|c|c|}
\hline & \multicolumn{4}{|c|}{ Terapia } & \\
\hline & & Cogniliva $n=18$ & $\begin{array}{c}\text { Activación } \\
\text { conductual } n=16\end{array}$ & Paroxetina $n=27$ & $\mathbf{P}$ \\
\hline \multirow{2}{*}{$\begin{array}{l}\text { Promedio de los puntajes a } \\
\text { las } 16 \text { semanas y desvío } \\
\text { estándar }\end{array}$} & Beck & $17,44(15,57)$ & $8,75(7,96)$ & $7,78(9,61)$ & 0,001 \\
\hline & Hamilton & $10,33(7,62)$ & $7,56(6,94)$ & $8,63(7,19)$ & 0,001 \\
\hline
\end{tabular}

\section{Conclusiones}

En pacientes con depresión severa, el TAC tiene similar eficacia que la paroxetina y mayor que la TC.

Fuente de financiamiento: Nacional Institute of Mental Health. Los autores trabajan como consultores $\mathrm{y} / \mathrm{o}$ disertantes de la industria farmacéutica incluyendo Glaxo Smith Kline.

\section{Comentario}

La DM tiene una prevalencia de 5 a $9 \%$ en mujeres y de 2 a $3 \%$ en hombres. El riesgo de padecerla a lo largo de la vida es de 10 a $25 \%$ en mujeres y de 5 a $12 \%$ en hombres'. Al ser un motivo de consulta frecuente en atención primaria ${ }^{2}$ es importante encontrar tratamientos efectivos, sencillos de aplicar, que no requieran un complejo entrenamiento y con pocos efectos adversos, principal motivo de falta de adherencia a la fármacoterapia ${ }^{3}$.

En este estudio no se observaron diferencias significativas en los pacientes con depresión leve, por lo que en estos casos sería aconsejable indicar tratamientos psicoterapéuticos, libres de efectos adversos.

En contraste con la farmacoterapia, el TAC retuvo un porcentaje mayor de pacientes en tratamiento. Los elementos que aporta explicarían su mejor desempeño respecto de la TC; en particular lo que se refiere al trabajo sobre los comportamientos evitativos, clásicamente estudiados en los trastornos de ansiedad. Los resultados de este trabajo sugieren que bloquear o detener la evitación sería clave para el tratamiento. Los pacientes aprenden a identificar patrones de evitación y a cambiarlos por una conducta de activación. EI TAC utiliza una aproximación al pensamiento negativo y rumiante, diferente del que usa la TC, ya que aquel enfatiza la atención sobre las consecuencias de la rumiación y los pensamientos negativos -entendidos como una estrategia de evitación y huida- y el uso alternativo de las estrategias de activación. Estos resultados cuestionan las guías de tratamientos clásicas para DM, en las que la TC era considerada tan efectiva como la farmacoterapia ${ }^{4,5,6}$. Vale aclarar que todavía no se conocen los resultados del TAC en el largo plazo.

\section{Conclusiones del comentador}

De confirmarse estos hallazgos, cabría desarrollar planes de entrenamiento y capacitación en TAC para aprovechar sus ventajas ya que sus intervenciones serían más simples y sus operadores más fácilmente entrenables ${ }^{7}$. Así, el tratamiento se podría adaptar mejor a poblaciones con menores recursos cognitivos de los necesarios para el abordaje cognitivo estándar.

Francisco Palacín [ Cátedra Clínica Psicológica, Psicoterapias, Emergencia e Inteconsulta II. Facultad de Psicología. Universidad de Buenos Aires.Director del Equipo Psy Adrogué. ]

Dimidjian S, Hollon SD, Dobson KS, Schmaling KB, Kohlenberg RJ, Addis ME, Gallop R, McGlinchey JB, Markley DK, Palacín. La terapia de activación conductual es tan eficaz como la medicación antidepresiva y superior a la terapia cognitiva, en la depresión mayor. Evid. actual. páct. ambul. 9(6);168. Nov-Dic. 2006. Comentado de: Dimidjian S et al. J Consult Clin Psychol. 2006; 74 (4): 658-670. PMID: 16881773

\section{Referencias}

1. American Psychiatric Association. Diagnostic and statistical on mental disorder (4th edition) (APA) 2000 Washinton DC. Author

2. Garay, C, Fabrisin, J.Tratamientos de la depression con apoyo empírico y su posible combinación. Revista Argentina Clínica Psicológica. Vol XII N 1. Abril 2003.

3. Silva D, Krotter L, Palacín F. Factores cognitivos en la adherencia al tratamiento. 9no. Congreso Internacional de Psiquiatría AAP 2002.

4. Agency for Care Policy and Research (AHCPR) Detection and Diagnosis. Depression in Primary Care Volume 1. 1993 Clinical Practice Guideline. Number 5. Agency for Care Poli,cy and Research Treatment of Major Depression. Depression in Primary Care Volume 2. (AHCPR) 1993 Clinical Practice Guideline. Number 5 .

6. American Psychiatric Association Practice guidelines for the treatment of patients with major depressive disorder (revision) 2000 American Journal of Psychiatry, 157, 1-45.

7. Beckham EE, Leber WR. Handbook of depression. 1995. Second Edition. Guilford Press. 